\title{
RP-HPLC analysis of furosine and acid-soluble $\beta$-lactoglobulin to assess the heat load of extended shelf life milk samples in Austria
}

\author{
Helmut K. Mayer*, Bernd Raba, Johannes Meier, Anita Schmid \\ Department of Food Science and Technology, Division of Food Chemistry, \\ BOKU - University of Natural Resources and Applied Life Sciences Vienna, Gregor Mendel-Strasse 33, \\ 1180 Vienna, Austria
}

Received 29 May 2009 - Revised 28 September 2009 - Accepted 26 October 2009

Published online 9 February 2010

\begin{abstract}
Recent trends to extend the shelf life of pasteurized milk, without the negative flavour normally associated with ultra-high-temperature (UHT) milk, have resulted in the development of extended shelf life (ESL) milk. Acid-soluble $\beta$-lactoglobulin $(\beta-\mathrm{Lg})$ and furosine contents were chosen as relevant indicators for heat load of ESL milk products. RP-HPLC methods were developed using the same column (Symmetry 300 ${ }^{\mathrm{TM}}$, Waters), which enabled the separation of whey proteins within $22 \mathrm{~min}$; furosine was analysed using ion-pair RP-HPLC within $8 \mathrm{~min}$. Electrophoresis was used as a high-throughput and cost-effective screening method to assess the impact of thermal processes on milk and to distinguish different categories of heat-treated milk samples. Liquid milk samples $(n=128$; including 7 raw, 33 pasteurized, 71 ESL, and 17 UHT milk samples) were obtained from retail outlets in Austria and analysed. Only $45 \%$ of the analysed samples designated as ESL milk showed furosine contents $<40 \mathrm{mg} \cdot 100 \mathrm{~g}^{-1}$ protein as well as acid-soluble $\beta$-Lg contents $>1800 \mathrm{mg} \cdot \mathrm{L}^{-1}$ milk, which had been discussed as threshold levels for ESL milk. A further 55\% of the analysed ESL milk samples had low acid-soluble $\beta$ - $\operatorname{Lg}\left(<500 \mathrm{mg} \cdot \mathrm{L}^{-1}\right)$ and high furosine contents $\left(>40 \mathrm{mg} \cdot 100 \mathrm{~g}^{-1}\right.$ protein), levels comparable to the excessive heat load of UHT milk. Thus, there is an urgent need for an EU regulation to define legal limits for the tolerable heat load of ESL milk as soon as possible.
\end{abstract}

extended shelf life milk / $\beta$-lactoglobulin / furosine / RP-HPLC / electrophoresis

摘要 - RP-HPLC 法测定糠氨酸和酸溶性 $\beta$-乳球蛋白及推测澳大利亚 ESL 乳的热负荷。近年 来, 长保质期的巴氏杀菌乳 (ESL) 由于其避免了 UHT 乳风味差的优点而得到了广泛的推 广。本文采用反相-高效液相色谱法测定乳中糠氨酸和 $\beta$-乳球蛋白含量, 以此推测 ESL 乳的 热负荷。HPLC 法可以在 $22 \mathrm{~min}$ 内有效地分离乳清蛋白, 而采用反相离子对色谱法可以在 $8 \mathrm{~min}$ 内分析糠氨酸。电泳法作为一种高通量和有效的检测方法可以评价热处理对乳的影 响和区分不同类型热处理的乳样品。取 128 个零售的乳样品 (其中 3 个生鲜乳、33 个 巴氏杀菌乳、71 个ESL 乳、17 个 UHT 乳), 根据分析其糠氨酸和 $\beta$-乳球蛋白含量来推测 热负荷。在 ESL 乳中, $45 \%$ 样品中每 100 克乳蛋白的糠氨酸含量低于 $40 \mathrm{mg}$, 每升乳 中 $\beta-\mathrm{Lg}$ 含量高于 $1800 \mathrm{mg}$, 这两个值低于 ESL 奶热负荷极限值。而另外 $55 \%$ 样品则有较 高的糠氨酸含量 (>40 mg.100 g 蛋白) 和较低的 $\beta-\mathrm{Lg}$ 含量 $\left(<500 \mathrm{mg} \cdot \mathrm{L}^{-1}\right)$, 这一数值与 UHT 乳的热负荷相当。因此, 有必要建议欧盟尽快制定法规来定义 ESL 乳的热负荷极限。

\section{长保质期乳 (ESL) / $\beta$-乳球蛋白 / 棣氨酸 / 反相-高效液相色谱 / 电泳}

*Corresponding author (通讯作者): helmut.mayer@boku.ac.at 
Résumé - Analyse de la furosine et de la $\beta$-lactoglobuline soluble à $\mathrm{pH}$ acide par RP-HPLC pour évaluer la charge thermique de laits de longue conservation en Autriche. La tendance actuelle visant à allonger la durée de conservation du lait pasteurisé, sans avoir les défauts de flaveur habituellement associés au lait UHT, a conduit au développement du lait de longue conservation ESL. Les concentrations en $\beta$-lactoglobuline soluble à $\mathrm{pH}$ acide $(\beta-\mathrm{Lg})$ et en furosine ont été choisies comme indicateurs pertinents de la charge thermique des produits laitiers ESL. Des méthodes RPHPLC ont été développées avec la même colonne (Symmetry 300 ${ }^{\mathrm{TM}}$, Waters), qui a permis de séparer les protéines sériques en $22 \mathrm{~min}$; la furosine était analysée par RP-HPLC en paire d'ions en 8 min. L'électrophorèse a été utilisée comme méthode économique de criblage à haut débit pour évaluer l'impact des traitements thermiques sur le lait et pour distinguer différentes catégories d'échantillons de lait traité thermiquement. Les échantillons de lait liquide $(n=128$; comprenant 7 échantillons de lait cru, 33 pasteurisé, 71 ESL et 17 UHT) ont été prélevés dans des points de vente en Autriche et analysés pour leur teneur en $\beta$-lactoglobuline et furosine. Seulement $45 \%$ des échantillons analysés désignés comme lait ESL présentaient des teneurs en furosine inférieures à $40 \mathrm{mg} \cdot 100 \mathrm{~g}^{-1}$ de protéine et des teneurs en $\beta$-lactoglobuline soluble à $\mathrm{pH}$ acide supérieures à $1800 \mathrm{mg} \cdot \mathrm{L}^{-1}$, valeurs qui ont été discutées pour être retenues comme niveaux seuils pour le lait ESL. D'autre part, $55 \%$ des échantillons de lait ESL analysés avaient des teneurs basses en $\beta$-lactoglobuline soluble à $\mathrm{pH}$ acide $\left(<500 \mathrm{mg} \cdot \mathrm{L}^{-1}\right)$ et élevées en furosine $\left(>40 \mathrm{mg} \cdot 100 \mathrm{~g}^{-1}\right.$ de protéine), ces niveaux étant comparables à ceux obtenus pour du lait UHT à charge thermique excessive. En conséquence, il existe un besoin urgent de réglementation au niveau européen pour définir au plus vite les limites obligatoires en matière de charge thermique tolérable pour le lait de longue conservation ESL.

lait de longue conservation / $\beta$-lactoglobuline / furosine / RP-HPLC / électrophorèse

\section{INTRODUCTION}

The relatively short shelf life of pasteurized milk has resulted in the development of ultra-high-temperature (UHT) treated milk for ambient distribution, which has gained widespread acceptance in many countries. In other areas, however, consumers have not accepted UHT milk because of the perceived "cooked" taste of the product. Consequently, the need for extending the shelf life of pasteurized milk, without the negative flavour change normally associated with UHT, has resulted in the development of milk products with taste similar to pasteurized milk, but with some of the obvious benefits of longer keeping ability of the product. The currently used methods to produce extended shelf life (ESL) milk are microfiltration, direct heat treatment such as injection or infusion (e.g. $127^{\circ} \mathrm{C}$ for 2-3 s), or in many cases also indirect heat treatment (e.g. $125^{\circ} \mathrm{C}$ for $2 \mathrm{~s}$ ) [15, $18,24,25]$. However, heating causes a significant loss of organoleptic and nutritional quality (e.g. vitamin destruction, precipitation of calcium phosphate, denaturation of whey proteins, and Maillard reaction) $[2,8,10,11,22]$. Furthermore, an undesirable precipitation of denatured proteins and minerals can be formed on the walls of heat exchangers [15, 18, 25].

To quantify the impact of thermal processes on milk, time temperature integrators (TTIs) can be used for heat-load evaluation. Several milk compounds have been suggested as potential TTIs for the assessment of heat treatment of milk (e.g. the enzymes alkaline phosphatase and lactoperoxidase; the whey protein $\beta$-lactoglobulin $\beta$-Lg; hydroxymethylfurfural, HMF; lactulose; and furosine) [3-6, 20, 22]. Type I reactions include the denaturation, degradation, and inactivation of heat-labile components (mainly whey proteins, enzymes, and vitamins) - these indicators are most suitable tools for the evaluation of low-heat treatments, whereas type II reactions include 
the formation of substances that are (almost) not present in unprocessed milk (e.g. lactulose, HMF, and furosine) - these indicators are more effective for the assessment of processes involving high temperatures $[3,4,22]$.

The quantitative determination of acid-soluble $\beta-\mathrm{Lg}$ has been proposed to distinguish between different categories of heat-treated milk. A minimum content of $2600 \mathrm{mg} \cdot \mathrm{L}^{-1}$ for pasteurized milk, of $2000 \mathrm{mg} \cdot \mathrm{L}^{-1}$ for high-pasteurized milk, and of $50 \mathrm{mg} \cdot \mathrm{L}^{-1}$ for UHT milk is within the limits proposed by the International Dairy Federation. In addition, furosine has also been proposed as a useful index for heat-induced changes in milk products. A furosine content of $8 \mathrm{mg} \cdot 100 \mathrm{~g}^{-1}$ protein has been suggested as upper limit for pasteurization, of $20 \mathrm{mg} \cdot 100 \mathrm{~g}^{-1}$ protein for high pasteurization, and of $250 \mathrm{mg} \cdot 100 \mathrm{~g}^{-1}$ protein for UHT processing $[3,4,6]$. At present, for lack of obligatory limits regarding the heat load of ESL milk in Europe, some rather general recommendations are circulating $\left(\beta-\mathrm{Lg}>1800 \mathrm{mg} \cdot \mathrm{L}^{-1}\right.$; furosine $<12 \mathrm{mg} \cdot 100 \mathrm{~g}^{-1}$ protein; and lactulose $\left.<30 \mathrm{mg} \cdot \mathrm{L}^{-1}\right)[9,15]$, which are taken seriously by individual dairy companies, but are neglected or completely ignored by all the others without any consequences by the control authorities. Moreover, as this "new" category of liquid milk has to be cooled also at $<6{ }^{\circ} \mathrm{C}$, pasteurized milk is currently being displaced in all supermarkets by ESL milk products. However, because of the partly changed nutritional and sensory quality of these products, this recent development towards an increasing consumption of milk "ESL" may have minor consequences for human nutrition in future.

The objective of this study was to improve RP-HPLC methods for the analysis of furosine and acid-soluble $\beta$ - $\mathrm{Lg}$ in milk using the same column and to determine the heat load of different categories of heat-treated liquid milk samples taken from retail outlets in Austria. Moreover, electrophoresis of acid-soluble whey proteins was used to assess the impact of thermal processes on market milk.

\section{MATERIALS AND METHODS}

\subsection{Milk samples}

Commercial liquid milk samples $(n=128)$ from different categories of heat treatment produced by different dairy companies were taken from retail outlets in Austria (see Tab. I). Samples of raw milk $(n=7)$, pasteurized milk $(n=33)$, ESL milk labelled with "länger frisch" (i.e. longer fresh) $(n=71)$, and UHT milk $(n=17)$ were aliquoted and kept frozen until consecutive chromatographic and electrophoretic analyses.

\subsection{RP-HPLC analysis of acid-soluble $\beta$-lactoglobulin}

Quantitative determination of acid-soluble $\beta$-lactoglobulin in liquid milk samples was performed using RP-HPLC following the IDF standard [14] with some modifications. Briefly, caseins and denatured whey proteins were precipitated at $\mathrm{pH} 4.6$ by the dropwise addition of $2 \mathrm{~mol} \cdot \mathrm{L}^{-1} \mathrm{HCl}$. Acid whey containing the acid-soluble whey proteins soluble at $\mathrm{pH} 4.6$ was separated by centrifugation and diluted (1:10 or 1:5 in the case of UHT milk) with sodium phosphate buffer solution (100 mmol. $\mathrm{L}^{-1}, \mathrm{pH}$ 6.7). Samples were filtered through $0.20 \mu \mathrm{m}$ Minisart RC 4 filters (Sartorius, Goettingen, Germany).

RP-HPLC was performed on a Waters chromatography system using a model $600 \mathrm{E}$ multisolvent delivery system, a Rheodyne 7725i injector, guard column (Sentry Guard, Symmetry ${ }^{\mathrm{TM}} \mathrm{C}_{18}, 3.5 \mu \mathrm{m}$, $2.1 \times 10 \mathrm{~mm}$ ), and a Symmetry ${ }^{\mathrm{TM}} 300$ $\mathrm{C}_{18}$ column $(3.5 \mu \mathrm{m}, \quad 2.1 \times 150 \mathrm{~mm})$ (Waters Corporation, Milford, MA, USA). 
Table I. Acid-soluble $\beta$ - $\mathrm{Lg}\left(\mathrm{mg} \cdot \mathrm{L}^{-1}\right)$ and furosine $\left(\mathrm{mg} \cdot 100 \mathrm{~g}^{-1}\right.$ protein) contents in different categories of heat-treated milk samples taken from retail outlets in Austria $(n=128)$. Results are given in order of decreasing $\beta$ - $\operatorname{Lg}$ contents of milk samples within each category.

\begin{tabular}{|c|c|c|c|c|c|c|}
\hline Code & Expiry date & Brand $^{1}$ & $\begin{array}{l}\text { Fat }^{2} \\
(\%)\end{array}$ & $\begin{array}{l}\text { Protein } \\
(\%)\end{array}$ & $\begin{array}{c}\text { Furosine } \\
\left(\mathrm{mg} \cdot 100 \mathrm{~g}^{-1} \text { protein }\right)\end{array}$ & $\begin{array}{c}\beta-\mathrm{Lg} \\
\left(\mathrm{mg} \cdot \mathrm{L}^{-1}\right) \\
\end{array}$ \\
\hline \multicolumn{7}{|c|}{ Raw milk samples $(n=7)$} \\
\hline 123 & 11.01.2009 & $\mathbf{A}$ & Nat. $^{3}$ & 3.41 & 5.9 & 5215 \\
\hline 125 & 11.01.2009 & B & Nat. $^{3}$ & 4.43 & 8.7 & 5210 \\
\hline 47 & 04.04 .2008 & $\mathbf{C}$ & Nat. $^{3}$ & 3.61 & 10.0 & 3752 \\
\hline 101 & 11.05 .2008 & $\mathbf{D}$ & 3.6 & 2.93 & 7.6 & 3706 \\
\hline 59 & 13.04.2008 & D & 3.6 & 3.13 & 7.0 & 3620 \\
\hline 100 & 02.05 .2008 & $\mathbf{C}$ & Nat. $^{3}$ & 3.21 & 8.4 & 3367 \\
\hline 62 & 11.04 .2008 & C & Nat. $^{3}$ & 3.24 & 8.5 & 3261 \\
\hline \multicolumn{7}{|c|}{ Pasteurized milk samples $(n=33)$} \\
\hline 9 & 11.12 .2007 & $\mathbf{E}$ & 3.5 & 3.71 & 10.2 & 3799 \\
\hline 80 & 22.04 .2008 & $\mathbf{F}$ & 1.8 & 3.29 & 10.3 & 3635 \\
\hline 56 & 11.12 .2007 & $\mathbf{F}$ & 3.5 & 3.57 & 10.0 & 3606 \\
\hline 45 & 09.04 .2008 & $\mathbf{E}$ & 3.5 & 3.18 & 9.6 & 3443 \\
\hline 129 & 19.12.2008 & G & 3.6 & 3.18 & 9.7 & 3429 \\
\hline 85 & 28.04 .2008 & $\mathbf{H}$ & 3.6 & 3.30 & 8.6 & 3418 \\
\hline 131 & 19.01.2009 & $\mathbf{G}$ & 3.6 & 3.21 & 9.5 & 3417 \\
\hline 106 & 13.05 .2008 & I & 1.5 & 3.09 & 9.5 & 3358 \\
\hline 84.3 & 29.04 .2008 & $\mathbf{J}$ & 3.5 & 3.28 & 10.0 & 3357 \\
\hline 84.1 & 27.04.2008 & $\mathbf{F}$ & 3.5 & 3.34 & 8.4 & 3355 \\
\hline 107 & 13.05.2008 & I & 3.8 & 3.03 & 10.3 & 3332 \\
\hline 105 & 12.05 .2008 & I & 1.5 & 3.14 & 9.2 & 3329 \\
\hline 99 & 09.05.2008 & $\mathbf{F}$ & 3.5 & 3.28 & 9.7 & 3325 \\
\hline 93 & 06.05 .2008 & $\mathbf{K}$ & 3.5 & 3.33 & 13.3 & 3305 \\
\hline 58 & 12.04 .2008 & $\mathbf{L}$ & 3.5 & 3.20 & 11.3 & 3301 \\
\hline 108 & 16.05 .2008 & $\mathbf{M}$ & 3.5 & 3.31 & 9.2 & 3280 \\
\hline 69 & 17.04 .2008 & $\mathbf{N}$ & 3.5 & 3.17 & 9.6 & 3108 \\
\hline 120 & 06.06 .2008 & $\mathbf{N}$ & 3.5 & 3.25 & 8.3 & 3082 \\
\hline 113 & 12.05 .2008 & $\mathbf{N}$ & 3.5 & 3.25 & 8.7 & 3061 \\
\hline 114 & 22.05 .2008 & $\mathbf{N}$ & 3.5 & 3.24 & 10.4 & 3052 \\
\hline 35 & 31.03 .2008 & $\mathbf{O}$ & 3.5 & 3.22 & 9.7 & 3049 \\
\hline 130 & 11.01 .2009 & G & 3.6 & 3.18 & 9.9 & 3045 \\
\hline 118 & 31.05 .2008 & $\mathbf{F}$ & 3.5 & 3.21 & 8.1 & 3041 \\
\hline 55 & 10.12 .2007 & G & 3.8 & 3.16 & 12.3 & 3025 \\
\hline 104 & 09.05.2008 & $\mathbf{O}$ & 3.5 & 3.04 & 8.8 & 2989 \\
\hline 54 & 10.12 .2007 & G & 1.6 & 3.40 & 9.8 & 2951 \\
\hline 111 & 16.05 .2008 & G & 1.6 & 3.32 & 10.8 & 2937 \\
\hline 78 & 21.04 .2008 & $\mathbf{P}$ & 3.5 & 3.39 & 11.0 & 2858 \\
\hline 14 & 29.02 .2008 & G & 4.5 & 3.06 & 10.9 & 2823 \\
\hline 116 & 23.05.2008 & $\mathbf{P}$ & 3.5 & 3.25 & 10.0 & 2748 \\
\hline 115 & 23.05.2008 & G & Nat. $^{3}$ & 3.14 & 8.8 & 2739 \\
\hline 121 & 07.06 .2008 & $\mathbf{P}$ & 3.5 & 3.38 & 10.2 & 2697 \\
\hline 33 & 31.03 .2008 & $\mathbf{J}$ & 3.5 & 3.32 & 11.4 & 2528 \\
\hline \multicolumn{7}{|c|}{ ESL milk samples $(n=71)$} \\
\hline 88 & 06.05 .2008 & $\mathbf{Q}$ & 0.5 & 3.30 & 11.7 & 3679 \\
\hline 95 & 19.05 .2008 & $\mathbf{R}$ & 3.5 & 3.42 & 11.0 & 3523 \\
\hline 94 & 09.05.2008 & $\mathbf{R}$ & 3.5 & 3.36 & 12.0 & 3501 \\
\hline
\end{tabular}


Table I. Continued.

\begin{tabular}{|c|c|c|c|c|c|c|}
\hline Code & Expiry date & Brand $^{1}$ & $\begin{array}{l}\mathrm{Fat}^{2} \\
(\%)\end{array}$ & $\begin{array}{c}\text { Protein } \\
(\%)\end{array}$ & $\begin{array}{c}\text { Furosine } \\
\left(\mathrm{mg} \cdot 100 \mathrm{~g}^{-1} \text { protein }\right)\end{array}$ & $\begin{array}{c}\beta-\mathrm{Lg} \\
\left(\mathrm{mg} \cdot \mathrm{L}^{-1}\right)\end{array}$ \\
\hline 87 & 10.05 .2008 & $\mathbf{Q}$ & 3.5 & 3.39 & 16.3 & 3433 \\
\hline 71 & 27.04 .2008 & M & 3.5 & 3.31 & 11.3 & 3371 \\
\hline 34 & 16.04 .2008 & $\mathbf{J}$ & 1.5 & 3.58 & 13.3 & 3246 \\
\hline 92 & 14.08 .2008 & $\mathbf{R}$ & 3.5 & 3.42 & 11.5 & 3223 \\
\hline 96 & 09.05 .2008 & $\mathbf{R}$ & 3.5 & 3.32 & 13.6 & 3125 \\
\hline 60 & 23.04 .2008 & $\mathbf{M}$ & 3.5 & 3.09 & 12.1 & 3088 \\
\hline 21 & 20.12 .2007 & $\mathbf{K}$ & 1.5 & 3.83 & 13.3 & 2866 \\
\hline 126 & 25.01 .2009 & $\mathbf{S}$ & 3.5 & 3.48 & 12.8 & 2858 \\
\hline 127 & 29.01 .2009 & $\mathbf{T}$ & 3.5 & 3.43 & 11.6 & 2792 \\
\hline 72 & 01.05 .2008 & $\mathbf{M}$ & 1.5 & 3.44 & 13.7 & 2663 \\
\hline 98 & 21.06 .2008 & $\mathbf{U}^{4}$ & 0.7 & 3.86 & 37.1 & 2653 \\
\hline 23 & 03.12 .2007 & $\mathbf{M}$ & 1.5 & 3.54 & 15.2 & 2541 \\
\hline 79 & 07.05 .2008 & $\mathbf{M}$ & 3.5 & 3.37 & 15.3 & 2533 \\
\hline 7.2 & 10.12 .2007 & $\mathbf{T}$ & 3.5 & 3.16 & 13.8 & 2475 \\
\hline 52 & 25.04 .2008 & $\mathbf{V}$ & 3.5 & 3.18 & 11.3 & 2431 \\
\hline 84.2 & 16.05 .2008 & $\mathbf{J}$ & 1.5 & 3.25 & 10.2 & 2406 \\
\hline 119 & 06.06 .2008 & $\mathbf{S}$ & 3.5 & 3.18 & 14.0 & 2404 \\
\hline 6 & 30.11 .2007 & $\mathbf{M}$ & 3.5 & 3.24 & 15.9 & 2402 \\
\hline 109 & 03.06 .2008 & $\mathbf{J}$ & 1.5 & 3.47 & 11.8 & 2390 \\
\hline 50 & 10.05 .2008 & $\mathbf{U}^{4}$ & 0.7 & 3.90 & 39.4 & 2311 \\
\hline 103 & 27.05 .2008 & $\mathbf{P}$ & 3.5 & 3.33 & 13.1 & 2270 \\
\hline 1 & 14.12 .2007 & $\mathbf{W}$ & 3.5 & 3.45 & 15.6 & 2259 \\
\hline 26 & 29.11 .2007 & $\mathbf{U}^{4}$ & 0.7 & 4.02 & 29.3 & 2245 \\
\hline 102 & 21.05 .2008 & $\mathbf{X}$ & 3.5 & 3.10 & 15.5 & 2180 \\
\hline 17 & 26.12.2007 & $\mathbf{Y}^{4}$ & 3.8 & 3.49 & 26.9 & 2025 \\
\hline 81 & 11.06 .2008 & $\mathbf{Y}^{4}$ & 3.8 & 3.17 & 17.3 & 1946 \\
\hline 49 & 08.04 .2008 & $\mathbf{S}$ & 3.5 & 3.32 & 24.5 & 1839 \\
\hline 18 & 11.03 .2008 & $\mathbf{S}$ & 3.5 & 3.32 & 23.6 & 1827 \\
\hline 42 & 07.05.2008 & $\mathbf{Y}^{4}$ & 3.8 & 3.26 & 22.5 & 1801 \\
\hline 86 & 02.05 .2008 & $\mathbf{Z}$ & 3.5 & 3.30 & 40.2 & 430 \\
\hline 12 & 15.03 .2008 & G & 1.0 & 3.14 & 50.5 & 401 \\
\hline 20 & 17.03.2008 & $\mathbf{F}^{5}$ & 1.5 & 3.28 & 123.9 & 387 \\
\hline 82 & 10.05 .2008 & $\mathbf{F}^{5}$ & 1.5 & 3.35 & 135.2 & 386 \\
\hline 28 & 16.03 .2008 & $\mathbf{F}$ & 3.5 & 3.30 & 41.4 & 379 \\
\hline 61 & 26.04 .2008 & $\mathbf{F}^{5}$ & 1.5 & 3.45 & 140.3 & 374 \\
\hline 73 & 04.05 .2008 & $\mathbf{F}$ & 3.5 & 3.13 & 40.8 & 336 \\
\hline 16 & 09.12 .2007 & $\mathbf{F}$ & 3.5 & 3.65 & 40.6 & 333 \\
\hline 124 & 02.02.2009 & $\mathbf{M}$ & 1.5 & 3.58 & 81.8 & 330 \\
\hline 13 & 16.03 .2008 & G & 3.5 & 3.21 & 49.3 & 328 \\
\hline 44 & 25.04 .2008 & G & 1.0 & 3.28 & 50.0 & 322 \\
\hline 2 & 05.12 .2007 & G & 1.0 & 3.39 & 47.6 & 306 \\
\hline 122 & 18.01.2009 & G & 3.6 & 3.19 & 49.8 & 300 \\
\hline 110 & 07.06 .2008 & G & 1.0 & 3.12 & 52.7 & 298 \\
\hline 112 & 02.06 .2008 & G & 3.6 & 3.00 & 53.3 & 291 \\
\hline 134 & 08.02 .2009 & G & 3.6 & 3.19 & 45.4 & 278 \\
\hline 31 & 13.12 .2007 & $\mathbf{R}$ & 0.1 & 3.8 & 74.7 & 260 \\
\hline 7.1 & 13.12 .2007 & $\mathbf{V}$ & 3.5 & 3.31 & 75.9 & 245 \\
\hline 51 & 25.04 .2008 & $\mathbf{R}$ & 0.1 & 3.67 & 82.4 & 240 \\
\hline 3 & 20.02 .2008 & $\mathbf{M}$ & 1.5 & 3.74 & 80.2 & 239 \\
\hline 22 & 09.12 .2007 & $\mathbf{M}$ & 3.5 & 3.39 & 72.0 & 234 \\
\hline
\end{tabular}


Table I. Continued.

\begin{tabular}{|c|c|c|c|c|c|c|}
\hline Code & Expiry date & Brand $^{1}$ & $\begin{array}{l}\mathrm{Fat}^{2} \\
(\%)\end{array}$ & $\begin{array}{c}\text { Protein } \\
(\%)\end{array}$ & $\begin{array}{c}\text { Furosine } \\
\left(\mathrm{mg} \cdot 100 \mathrm{~g}^{-1} \text { protein }\right)\end{array}$ & $\begin{array}{r}\beta-\mathrm{Lg} \\
\left(\mathrm{mg} \cdot \mathrm{L}^{-1}\right) \\
\end{array}$ \\
\hline 83 & 07.05 .2008 & M & 1.8 & 3.51 & 100.1 & 231 \\
\hline 137 & 25.01 .2009 & G & 1.0 & 3.27 & 50.3 & 230 \\
\hline 15 & 13.12 .2008 & $\mathbf{J}$ & 1.5 & 3.59 & 77.9 & 229 \\
\hline 132 & 09.01.2009 & G & 3.6 & 3.16 & 48.3 & 226 \\
\hline 74 & 03.05 .2008 & $\mathbf{P}$ & 3.5 & 3.17 & 94.2 & 226 \\
\hline 135 & 08.01.2009 & G & 1.0 & 2.98 & 46.9 & 217 \\
\hline 25 & 26.03 .2008 & M & 1.8 & 3.81 & 62.2 & 213 \\
\hline 24 & 18.03 .2008 & M & 1.8 & 3.66 & 46.2 & 212 \\
\hline 57 & 23.04 .2008 & $\mathbf{X}$ & 3.5 & 3.13 & 82.0 & 212 \\
\hline 11 & 13.03.2008 & V & 3.5 & 3.02 & 94.1 & 211 \\
\hline 19 & 17.03 .2008 & $\mathbf{M}$ & 0.9 & 3.45 & 95.1 & 206 \\
\hline 4 & 04.12 .2007 & $\mathbf{M}$ & 1.8 & 3.75 & 82.5 & 205 \\
\hline 77 & 08.05 .2008 & $\mathbf{M}$ & 1.8 & 3.58 & 74.2 & 204 \\
\hline 136 & 25.01 .2009 & G & 1.0 & 3.26 & 52.4 & 192 \\
\hline 133 & 26.01.2009 & G & 3.6 & 3.21 & 40.1 & 186 \\
\hline 76 & 06.05 .2008 & M & 0.9 & 3.42 & 92.5 & 184 \\
\hline 91 & 20.06 .2008 & $\mathbf{A A}^{4}$ & 0.1 & 3.79 & 262.2 & 160 \\
\hline 10 & 02.04 .2008 & $\mathbf{A A}^{4}$ & 0.1 & 4.15 & 225.5 & 140 \\
\hline \multicolumn{7}{|c|}{ UHT milk samples $(n=17)$} \\
\hline 41 & 18.04 .2008 & $\mathbf{M}^{5}$ & 1.8 & 3.50 & 284.7 & 403 \\
\hline 75 & 14.07.2008 & $\mathbf{R}$ & 3.5 & 3.39 & 159.2 & 344 \\
\hline 39 & 23.03.2008 & BB & 0.1 & 3.64 & 93.1 & 256 \\
\hline 36 & 28.05 .2008 & $\mathbf{R}$ & 0.5 & 3.48 & 160.3 & 244 \\
\hline 48 & 15.03.2008 & $\mathbf{E}$ & 0.5 & 3.66 & 151.6 & 232 \\
\hline 68 & 10.07 .2008 & $\mathbf{F}$ & 0.5 & 3.30 & 165.5 & 221 \\
\hline 43 & 14.06.2008 & $\mathbf{C C}^{5}$ & 1.5 & 3.44 & 352.4 & 220 \\
\hline 40 & 09.03 .2008 & $\mathbf{R}$ & 3.5 & 3.56 & 161.3 & 216 \\
\hline 66 & 06.06 .2008 & BB & 3.8 & 3.26 & 109.1 & 214 \\
\hline 38 & 17.05 .2008 & DD & 1.5 & 3.62 & 138.9 & 213 \\
\hline 117 & 15.07 .2008 & $\mathbf{R}$ & 0.5 & 3.55 & 168.1 & 196 \\
\hline 90 & 14.08 .2008 & $\mathbf{E E}$ & 0.5 & 3.53 & 137.8 & 183 \\
\hline 89 & 28.07.2008 & $\mathbf{E E}$ & 3.5 & 3.39 & 181.5 & 172 \\
\hline 8 & 02.03 .2008 & $\mathbf{R}$ & 0.5 & 3.21 & 162.8 & 170 \\
\hline 37 & 01.04 .2008 & $\mathbf{A} \mathbf{A}^{5}$ & 1.5 & 3.32 & 207.0 & 166 \\
\hline 97 & 20.08 .2008 & $\mathbf{F F}^{5}$ & 3.5 & 3.34 & 471.9 & 159 \\
\hline 67 & 22.07 .2008 & $\mathbf{F F}^{5}$ & 3.5 & 3.20 & 485.0 & 158 \\
\hline
\end{tabular}

${ }^{1}$ Bold values are anonymous designation of products.

${ }^{2}$ Fat content according to labelling.

${ }^{3}$ Natural fat content (min. $3.5 \%$ ).

${ }_{5}^{4}$ Protein-enriched milk sample.

${ }^{5}$ Lactose-free milk sample.

Column eluates were monitored at $205 \mathrm{~nm}$ using a Waters 2489 UV/VIS Detector interfaced with a PC running Waters Millennium ${ }^{32}$ chromatography manager. After flushing the column for $20 \mathrm{~min}$ with solvent B [0.1\% $(\mathrm{v} / \mathrm{v})$ trifluoroacetic acid (TFA) in acetoni- trile], the initial conditions were set until a stable baseline was observed ( $30 \mathrm{~min})$ : $64 \%$ solvent A $[0.1 \%(\mathrm{v} / \mathrm{v})$ TFA in ultra-high quality (UHQ) water] and 36\% solvent B. Samples $(10 \mu \mathrm{L})$ were applied in duplicate onto the column and eluted at $40{ }^{\circ} \mathrm{C}$ at a flow rate 
of $0.35 \mathrm{~mL} \cdot \mathrm{min}^{-1}$ using the following optimized gradient: from $36 \%$ to $50 \%$ solvent B linearly over $14 \mathrm{~min}$, increasing to $100 \% \mathrm{~B}$ within $0.5 \mathrm{~min}$, and finally holding at $100 \%$ B for $3.5 \mathrm{~min}$. The column was then subsequently returned to the initial conditions within $1 \mathrm{~min}$ and equilibrated for 15 min before the next sample injection. $\beta$-lactoglobulin with a purity of $\sim 90 \%$ (Sigma Chemical Co., St. Louis, MO, USA) was used as standard for calibration. Calibration curve $(0.2,0.4,0.8,1.6,2.4$, and $3.2 \mu \mathrm{g} \cdot 10 \mu \mathrm{L}^{-1}$ injection volume) was obtained by plotting peak area versus microgram of $\beta-\mathrm{Lg}$. Concentrations of $\beta-\mathrm{Lg}$ were displayed as microgram, which were subsequently converted to the results given in $\mathrm{mg} \cdot \mathrm{L}^{-1}$ milk (Tab. I).

\subsection{RP-HPLC analysis of furosine}

Although an IDF standard exists also for the determination of furosine content in milk [13], an ion-pair RP-HPLC method was developed to analyse furosine $[\varepsilon-N$ (2-furoylmethyl)-L-lysine] using the same column as for $\beta$-Lg. Sample preparation including acid hydrolysis and cleaning of hydrolysates using solid-phase extraction (SPE) prior to chromatographic separation was performed according to the IDF standard [13] with some modifications. Briefly, $2 \mathrm{~mL}$ milk sample (UHT milk was diluted 1:4) was hydrolysed in the presence of $6 \mathrm{~mL}$ of $10.6 \mathrm{~mol} \cdot \mathrm{L}^{-1} \mathrm{HCl}$ for $23 \mathrm{~h}$ at $110^{\circ} \mathrm{C}$ in screw-capped Pyrex tubes (after bubbling with nitrogen for $2 \mathrm{~min}$ ). The hydrolysate was filtered (Schleicher \& Schuell 5951/2) and applied to SPE to minimize contamination: $0.5 \mathrm{~mL}$ of hydrolysate was added to a pre-wetted $(5 \mathrm{~mL}$ methanol and $10 \mathrm{~mL}$ water) Sep-Pak ${ }^{\circledR}$ Vac $3 \mathrm{cc}$ (500 mg) $\mathrm{C}_{18}$ cartridge (Waters); the eluted liquid was discarded, and furosine was then eluted with $3 \mathrm{~mL}$ of $3 \mathrm{~mol} \cdot \mathrm{L}^{-1} \mathrm{HCl}$. In contrast to the IDF standard procedure [13], samples were not directly used for HPLC analyses, but were dried as reported by some authors [8, 16, 26]. Purified eluates $(200 \mu \mathrm{L})$ were gently vacuum-dried using a Waters Pico $\cdot$ Tag $^{\text {TM }}$ workstation, and dried samples were dissolved in $200 \mu \mathrm{L}$ of a freshly prepared mixture of water, acetonitrile, and formic acid (94.8:5:0.2) before HPLC analysis. Duplicate sample hydrolysates were used for drying.

The same HPLC equipment and in particular the same HPLC column was used as described for the analysis of $\beta-\operatorname{Lg}$ to avoid the need for recurrent replacement of column and to improve the flexibility of HPLC equipment. Samples were filtered through $0.20 \mu \mathrm{m}$ Minisart RC 4 filters (Sartorius). Column eluates were monitored at $280 \mathrm{~nm}$ using a Waters 2489 UV/VIS Detector. In contrast to other authors [7, 8, $16,26,27]$, two separated mobile phases were used, which were continuously mixed by the used HPLC multisolvent delivery system during isocratic separation of furosine. Solvent A consisted of $0.2 \%$ formic acid in $5 \mathrm{mmol} \cdot \mathrm{L}^{-1}$ sodium heptane sulphonate (Sigma) solution (prepared from a $50 \mathrm{mmol} \cdot \mathrm{L}^{-1}$ stock solution), and solvent B was $100 \%$ acetonitrile. After flushing the column for $20 \mathrm{~min}$ with solvent $\mathrm{B}$, the initial conditions were set until a stable baseline was observed $(\sim 30 \mathrm{~min}): 89 \%$ solvent $\mathrm{A}$ and $11 \%$ solvent B. Samples $(20 \mu \mathrm{L})$ were applied onto the column and eluted at $35{ }^{\circ} \mathrm{C}$ at a flow rate of $0.35 \mathrm{~mL} \cdot \mathrm{min}^{-1}$ using isocratic conditions. Intervals for sample injections were $20 \mathrm{~min}$, each sample was injected in duplicate. Furosine (NeoMPS PolyPeptide Laboratories Group, Strasbourg, France) was used as standard. Calibration $(5,10,20,40,80$, and $160 \mathrm{pmol} \cdot 20 \mu \mathrm{L}^{-1}$ injection volume) was performed by plotting peak area versus picomole of furosine. Concentrations of furosine were displayed as picomole, which were subsequently converted to the results given in $\mathrm{mg} \cdot 100 \mathrm{~g}^{-1}$ protein (Tab. I). As protein results were needed for the calculation, total 
nitrogen content of milk samples was determined using the Kjeldahl method after mineralization [12].

\subsection{SDS-PAGE of milk proteins}

Sodium dodecyl sulphate-polyacrylamide gel electrophoresis (SDS-PAGE) was used to analyse total milk proteins as well as acid-soluble whey proteins. Milk samples $(100 \mu \mathrm{L})$ and acid whey after isoelectric precipitation of casein and denatured whey proteins $(100 \mu \mathrm{L})$ were directly diluted (1:5) with sample buffer (2\% SDS in $50 \mathrm{mmol} \cdot \mathrm{L}^{-1}$ Tris- $\mathrm{HCl}$ buffer, $\mathrm{pH}$ 7.2), mixed with $5 \mu \mathrm{L} 2.6 \mathrm{~mol} \cdot \mathrm{L}^{-1}$ dithiothreitol (DTT) solution, and heated in a thermoblock for $10 \mathrm{~min}$ at $100{ }^{\circ} \mathrm{C}$. After cooling to room temperature, $5 \mu \mathrm{L}$ DTT solution was added as a reducing agent again. Samples were mixed with $10 \%(\mathrm{v} / \mathrm{v})$ bromophenol blue solution $(0.1 \%$ bromophenol blue and $8.7 \%$ glycerol), and proteins were separated by SDS-PAGE (15\% T) according to a method described recently [19].

\subsection{Alkaline PAGE of whey proteins}

Alkaline PAGE was used to separate acid-soluble whey proteins. Acid whey (see Sect. 2.2) $(100 \mu \mathrm{L})$ was directly diluted (1:5) with sample buffer $\left(10 \mathrm{mmol} \cdot \mathrm{L}^{-1}\right.$ Tris and $77 \mathrm{mmol} \cdot \mathrm{L}^{-1}$ glycine buffer, $\mathrm{pH} 8.3$ ) and mixed with $10 \%(\mathrm{v} / \mathrm{v})$ bromophenol blue solution. Native PAGE $(12.5 \%$ T) was performed using a dual cooled vertical slab gel electrophoresis unit SE 600 (Hoefer Scientific Instruments, San Francisco, CA, USA) as reported recently [19].

\section{RESULTS AND DISCUSSION}

\subsection{Electrophoresis of milk proteins}

In this study, electrophoresis of milk proteins was performed as a rapid and reliable screening technique to assess the heat load of milk and to distinguish different categories of heat-treated milk samples. The electrophoretic separation of the total milk proteins obtained by SDS-PAGE analysis of ESL milk samples was just to confirm that all milk samples analysed had almost the same electrophoretic pattern. After cooking milk samples (for $10 \mathrm{~min}$ at $100{ }^{\circ} \mathrm{C}$ ) in the presence of SDS and DTT, intense casein bands, strong $\beta$ - Lg, and weak $\alpha$-La bands were detected in all samples, irrespective of the heating processes during manufacturing (results not shown). However, SDS-PAGE patterns of the acid whey of ESL milk samples showed distinct differences between individual samples. Since precipitated casein (including also the heat-denatured whey proteins) had been removed by centrifugation, acid whey showed just the acid-soluble whey proteins soluble at $\mathrm{pH} 4.6$ (results not shown). In accordance with the results obtained by SDS-PAGE, the separation of non-denatured whey proteins by alkaline PAGE demonstrated even more clearly the differences between samples (Fig. 1). Figure 1 shows the electrophoretic patterns of selected milk samples from different categories of heat treatment (raw, pasteurized, ESL, and UHT milk). It is obvious that band intensity of acid-soluble whey proteins decreased corresponding to an increased heat load of milk samples analysed (from raw milk to UHT milk). The main whey proteins involved are in order of decreasing heat stability: $\alpha$-La $>\beta$-Lg $>$ BSA $>$ immunoglobulins $[1,4,17]$. Thus, depending on heat stability of whey proteins, individual whey protein fractions decrease as a consequence of heating processes and can be used as a reliable tool to study the heat load of commercial milk samples. All the more, considering the fact that costs for acetonitrile needed for HPLC analyses of acid-soluble $\beta-\mathrm{Lg}$ and furosine have tremendously increased during last months, electrophoresis proved to be a high-throughput, costeffective, and reliable screening method 


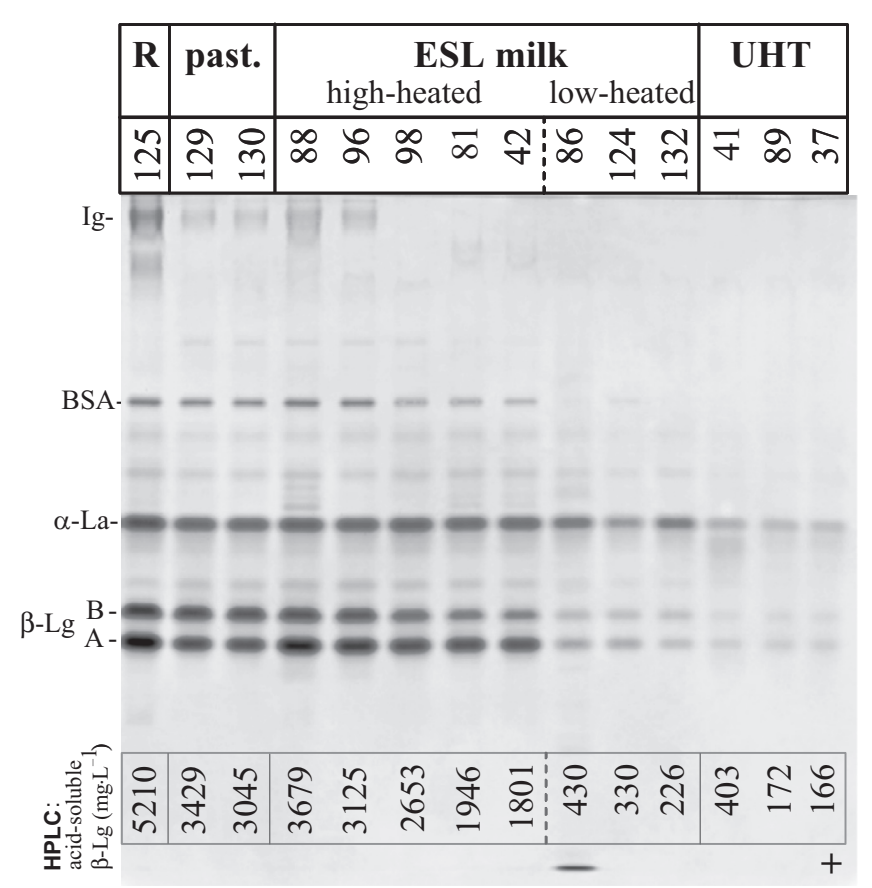

Figure 1. Alkaline polyacrylamide gel electrophoresis of whey protein fractions soluble at $\mathrm{pH} 4.6$ from different categories of heat-treated milk: raw milk (125), pasteurized milk (129 and 130), lowheated $(88,96,98,81$, and 42) and high-heated $(86,124$, and 132) ESL milk, and UHT milk (41, 89 , and 37) samples. Ig (immunoglobulins), BSA (bovine serum albumin), $\alpha$-lactalbumin ( $\alpha$-La), and $\beta$-lactoglobulin $(\beta-\mathrm{Lg})$ were separated depending on their negative charge.

to differentiate between low-heated and high-heated ESL milk.

\subsection{RP-HPLC analysis of acid-soluble $\beta$-lactoglobulin in milk samples}

As electrophoretic patterns of acidsoluble whey proteins were used just for screening purposes, an HPLC method for the quantitative determination of acidsoluble $\beta-\mathrm{Lg}$ was established. Figure 2 shows the RP-HPLC chromatogram of a standard mixture of $\alpha$-La and $\beta$-Lg using a Symmetry $300^{\mathrm{TM}} \mathrm{C}_{18}$ column (Waters), which enabled an acceptable resolution of whey proteins within $22 \mathrm{~min}$ superior to that reported in the IDF standard procedure [14]. Linearity of calibration was appropriate $\left(R^{2}=0.999\right)$ in the range of $0.2-3.2 \mu \mathrm{g}$, corresponding directly to the range of most analysed sample contents $\left(200-3200 \mathrm{mg} \cdot \mathrm{L}^{-1}\right.$ milk). The precision of the entire procedure including sample preparation and RP-HPLC analysis (same day) was evaluated on milk samples having low and high $\beta$-Lg contents. The relative standard deviation (RSD) was $1.91 \%$ obtained on a UHT milk sample with an average $\beta$ - Lg content of $339.9 \pm 6.5 \mathrm{mg} \cdot \mathrm{L}^{-1}$ milk, and $0.26 \%$ on an ESL milk sample $\left(2397.8 \pm 6.2 \mathrm{mg} \cdot \mathrm{L}^{-1}\right)$ $(n=8)$. Results of all milk samples analysed are given in Table I. The obvious difference between ESL milk samples of good and poor 
(a)
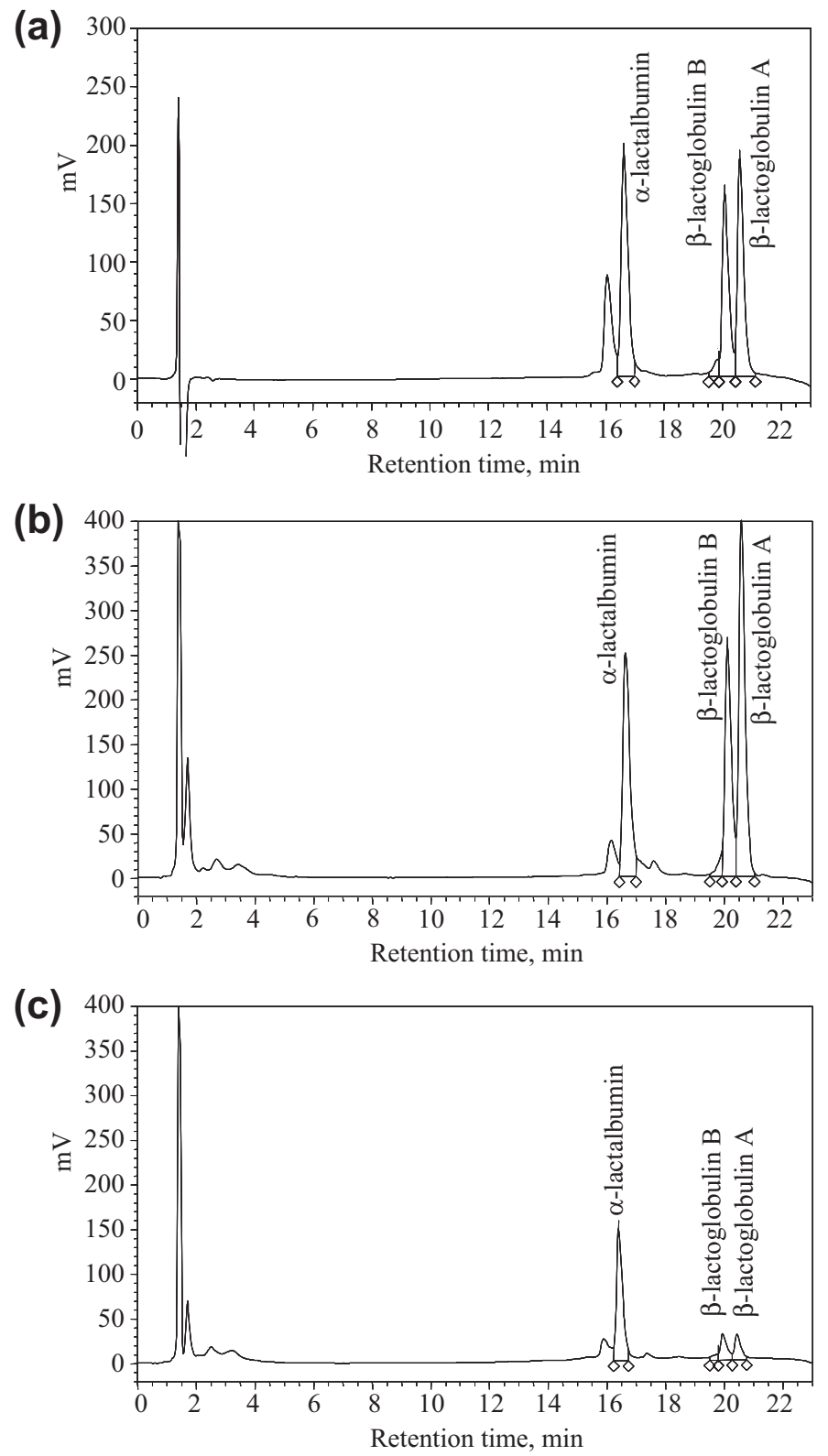

Figure 2. RP-HPLC chromatograms of a standard mixture of $\alpha$-lactalbumin $(0.8 \mu \mathrm{g})$ and $\beta$-lactoglobulin $(1.6 \mu \mathrm{g})$ per $10 \mu \mathrm{L}$ injection volume each (a), and of acid-soluble whey proteins in low-heated (b) and high-heated (c) ESL milk samples, having native $\beta$-Lg contents of 2792 and $260 \mathrm{mg} \cdot \mathrm{L}^{-1}$ milk, respectively (Nos. 127 and 31 ). 
quality is demonstrated in Figure 2. Compared to the results obtained by other authors $[14,21]$, a faster separation and a much more stable baseline were the most striking advantages of the HPLC column used in the present study.

\subsection{RP-HPLC analysis of furosine in milk samples}

A specific "Furosine dedicated" RPHPLC $\mathrm{C}_{8}$ column had been proposed by the FIL/IDF standard [13], whereas different types of $\mathrm{C}_{18}$ columns were reported for RP-HPLC by other authors [7, 8, 16, 23, $26,27]$. However, as furosine and $\beta$-Lg contents of many milk samples were to be analysed in alternating series, an effort was made to develop an RP-HPLC method for the analysis of furosine using the same column as for $\beta-\mathrm{Lg}$, to avoid recurrent replacement of column during this study. Figure 3 shows the chromatogram of a furosine standard using a Symmetry $300^{\mathrm{TM}} \mathrm{C}_{18}$ column (Waters), which enabled an excellent separation of furosine within $8 \mathrm{~min}$ superior to that reported in the IDF standard procedure within $22 \mathrm{~min}$ [13], and comparable to that of other authors $[8,16,26]$. However, in contrast to all available reports $[7,8,16,26,27]$, two separated mobile phases were used, which were continuously mixed by the used HPLC multisolvent delivery system during isocratic separation of furosine. Continuous mixing of two separated solvents (solvent A was $0.2 \%$ formic acid in $5 \mathrm{mmol} \cdot \mathrm{L}^{-1}$ sodium heptane sulphonate and solvent B was $100 \%$ acetonitrile) was of utmost importance to get a stable baseline, a proper resolution, and constant retention time of furosine peaks in different samples.

Linearity of regression line was appropriate $\left(R^{2}=0.999\right)$ in the range of 5-160 pmol. Precision of the entire procedure $(n=32)$ including acid hydrolysis $(n=4)$, Sep-Pak ${ }^{\circledR}$ purification $(n=2)$, vacuum-drying in Pico $\cdot$ Tag $^{\mathrm{TM}}$ workstation $(n=2)$, and
RP-HPLC analysis (two injections each) was evaluated on an ESL milk sample with an average furosine content of $49.87 \pm 0.55 \mathrm{mg} \cdot 100 \mathrm{~g}^{-1}$ protein $(\mathrm{RSD}$ was $1.10 \%)$. Results of all milk samples analysed are given in Table I. The substantial difference between ESL milk samples of good and poor quality is shown in Figure 3.

\subsection{Comparison of acid-soluble $\beta-\mathrm{Lg}$ and furosine contents in ESL milk samples}

Acid-soluble $\beta$ - $\operatorname{Lg}$ and furosine contents of all milk samples from different categories of heat treatment (raw, pasteurized, ESL, and UHT milk) analysed in this study are listed in Table I. Results were given in order of decreasing $\beta$-Lg contents of milk samples within each category, mean values for each category are compiled in Figure 4. As expected, pasteurized milk samples had high $\beta$-Lg (mean value $=3177 \pm 288 \mathrm{mg} \cdot \mathrm{L}^{-1}$ ) and low furosine $\left(9.9 \pm 1.3 \mathrm{mg} \cdot 100 \mathrm{~g}^{-1}\right.$ protein) contents, whereas UHT milk conversely showed very low $\beta-\mathrm{Lg}\left(226 \pm 67 \mathrm{mg} \cdot \mathrm{L}^{-1}\right)$ and high furosine $\left(204 \pm 124 \mathrm{mg} \cdot 100 \mathrm{~g}^{-1}\right.$ protein) contents. Surprisingly, ESL milk samples had to be divided into two separate groups: ESL milk of good quality (45\% of analysed samples) showed acid-soluble $\beta$-Lg contents $>1800 \mathrm{mg} \cdot \mathrm{L}^{-1}$ and furosine contents $<40 \mathrm{mg} \cdot 100 \mathrm{~g}^{-1}$ protein, whereas ESL milk of poor quality had low acidsoluble $\beta$ - $\mathrm{Lg}\left(<500 \mathrm{mg} \cdot \mathrm{L}^{-1}\right)$ and high furosine contents $\left(>40 \mathrm{mg} \cdot 100 \mathrm{~g}^{-1}\right.$ protein), which was comparable to the excessive heat load of UHT milk (Fig. 4). This remarkable bisection of ESL milk samples can be seen even more clearly in Figure 5, where $\beta$-Lg contents of all analysed milk samples were plotted against furosine contents $\left(R^{2}=0.856\right)$. Raw milk, pasteurized, and ESL milk samples of good quality were clustered on top and on the left side (having low furosine and high $\beta$-Lg contents), whereas ESL milk samples of poor quality and UHT milk samples were grouped on the bottom 

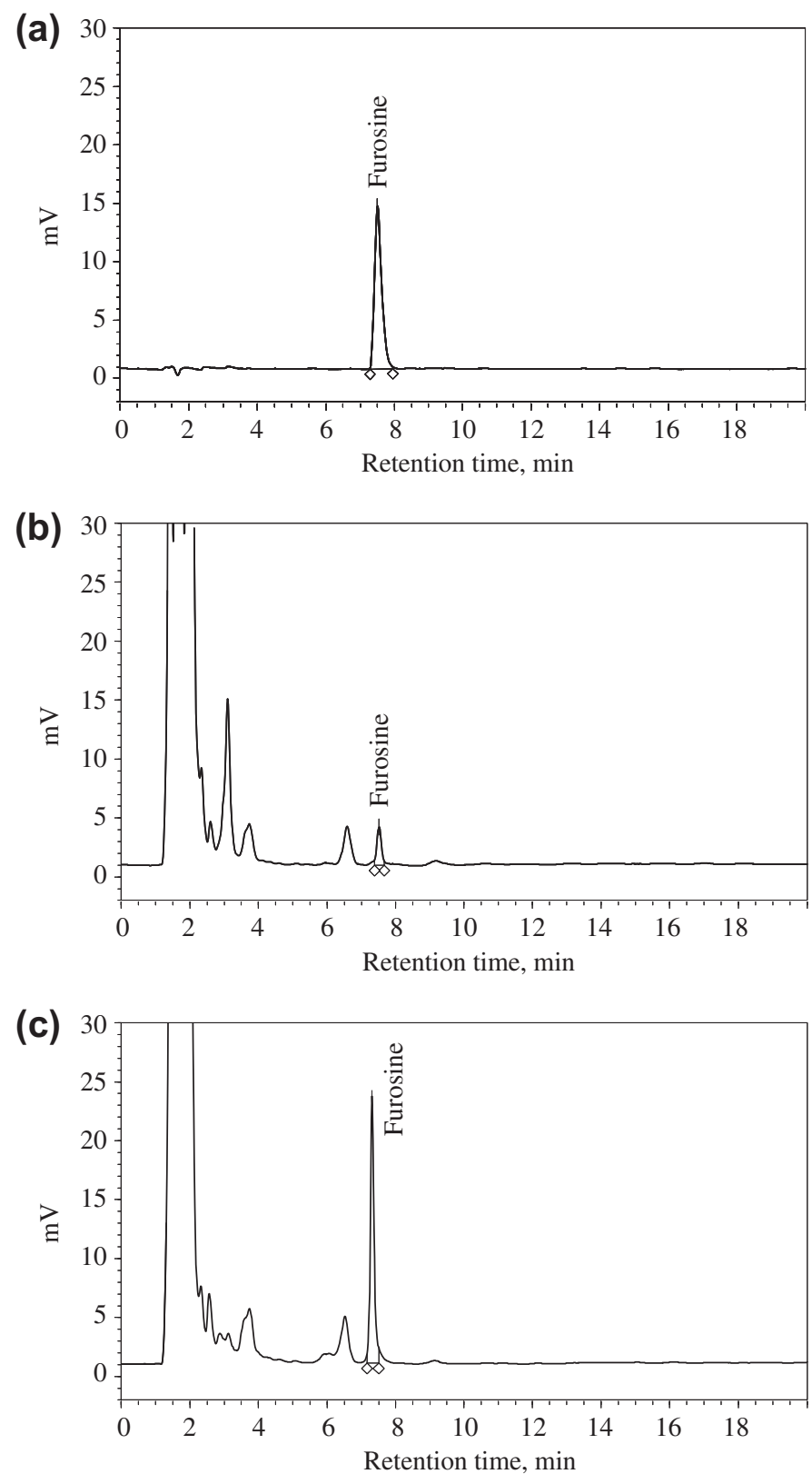

Figure 3. RP-HPLC chromatograms of a furosine standard with 80 pmol per $20 \mu \mathrm{L}$ injection volume (a), and of furosine in low-heated (b) and high-heated (c) ESL milk samples, having furosine contents of 11.6 and $74.7 \mathrm{mg} \cdot 100 \mathrm{~g}^{-1}$ protein, respectively (Nos. 127 and 31). 


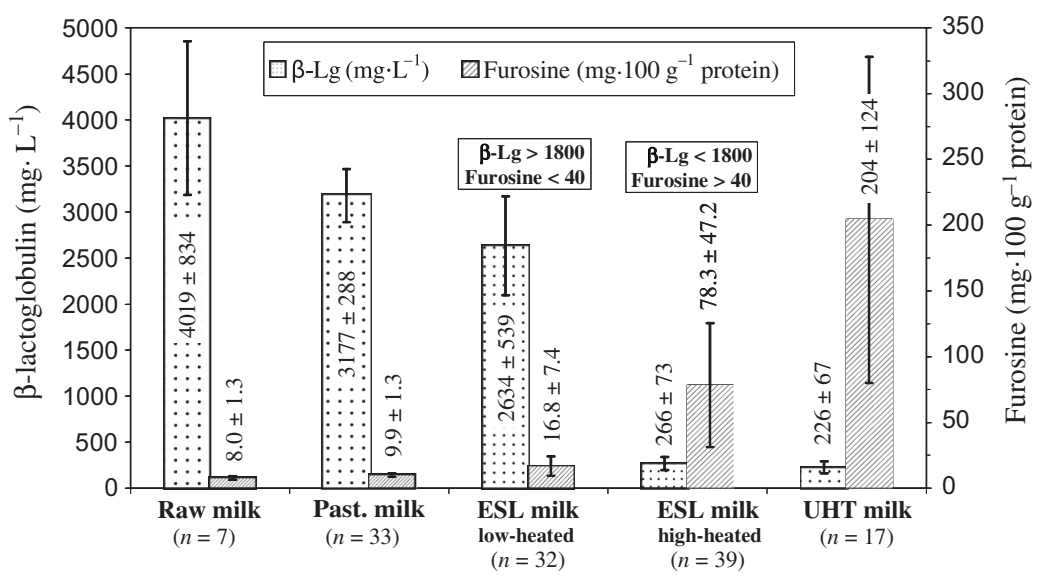

Figure 4. Mean values $( \pm \mathrm{SD})$ for acid-soluble $\beta$ - $\mathrm{Lg}\left(\mathrm{mg} \cdot \mathrm{L}^{-1}\right)$ and furosine $\left(\mathrm{mg} \cdot 100 \mathrm{~g}^{-1}\right.$ protein) contents in different categories of heat-treated milk samples taken from retail outlets in Austria $(n=128)$.

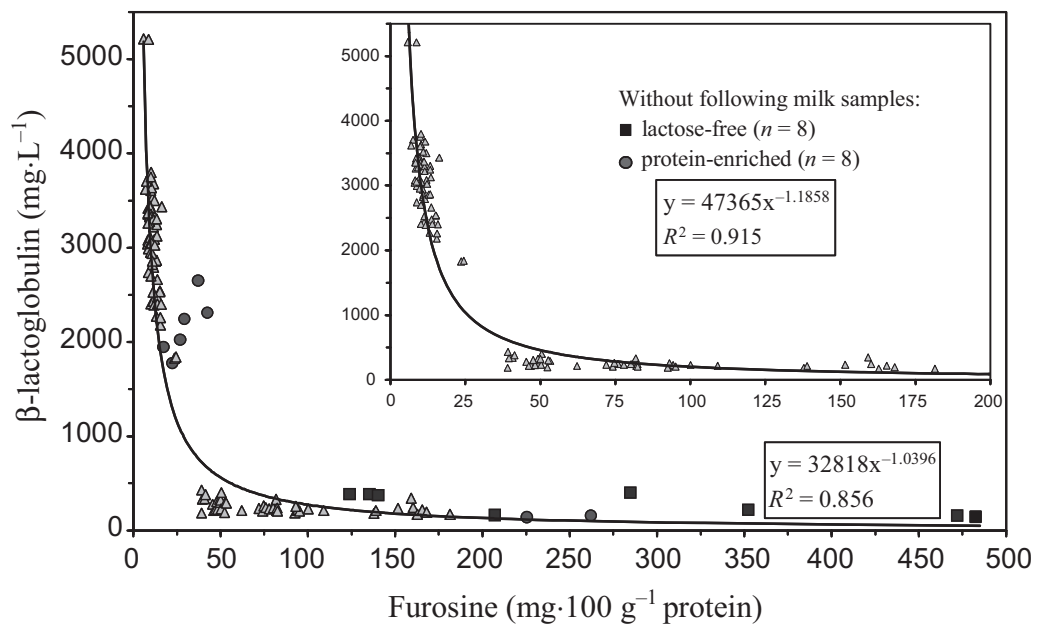

Figure 5. Relationship between acid-soluble $\beta-\mathrm{Lg}\left(\mathrm{mg} \cdot \mathrm{L}^{-1}\right)$ and furosine ( $\mathrm{mg} \cdot 100 \mathrm{~g}^{-1}$ protein) contents of milk samples taken from retail outlets in Austria $(n=128)$.

and on the right side of the graph (showing high furosine and low $\beta$-Lg contents). As protein-enriched and lactose-free milk samples partly did not fit the curve as perfect as the other samples, they were omitted to get a logarithmic relationship with an improved regression coefficient $\left(R^{2}=0.915\right)$.

Figure 6 shows the relative distribution of acid-soluble $\beta-\mathrm{Lg}$ and furosine contents of all ESL milk samples analysed in this study $(n=71)$. ESL milk samples of good quality $\left(\beta-\mathrm{Lg}>1800 \mathrm{mg} \cdot \mathrm{L}^{-1} ; 45 \%\right)$ were subdivided into four groups, taking into account some additional limits of 2000 , 2500 , and $3000 \mathrm{mg} \cdot \mathrm{L}^{-1}$. A minimum content of $2000 \mathrm{mg} \cdot \mathrm{L}^{-1}$ had been proposed for high-pasteurized milk [3, 4], whereas $1800 \mathrm{mg} \cdot \mathrm{L}^{-1}$ was discussed as threshold 

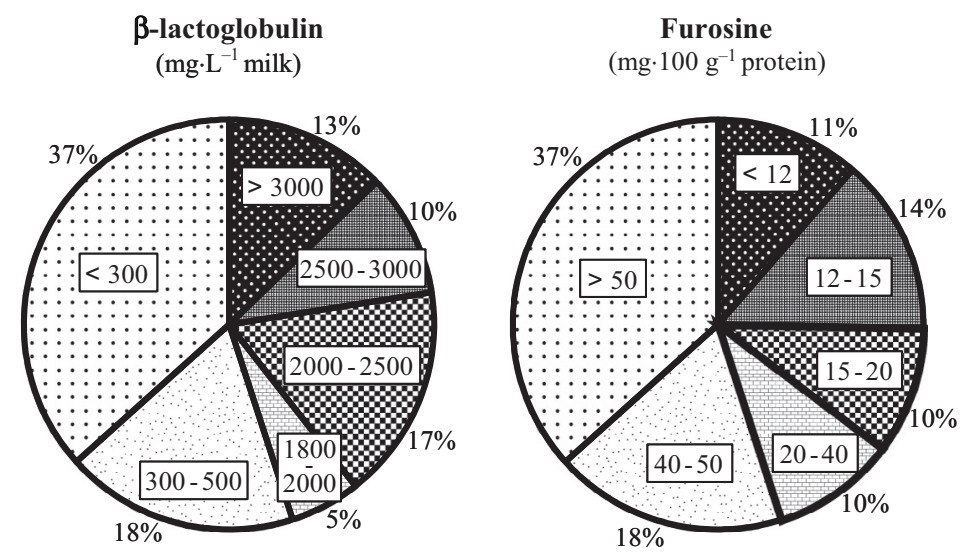

Figure 6. Relative distribution of acid-soluble $\beta-\mathrm{Lg}\left(\mathrm{mg} \cdot \mathrm{L}^{-1}\right)$ and furosine $\left(\mathrm{mg} \cdot 100 \mathrm{~g}^{-1}\right.$ protein) contents in ESL milk samples taken from retail outlets in Austria $(n=71)$.

level in Austria and in some other European countries due to the lack of obligatory limits regarding the heat load of ESL milk $[9,15]$. As there were only four samples $(5 \%)$ between these two suggested limits (Tab. I and Fig. 6), both would be appropriate as obligatory limits to control the heat load of ESL milk samples by regulatory authorities.

The relative distribution of furosine contents of all ESL milk samples reflected the above-mentioned remarkable bisection of ESL milk samples of different quality in a very similar manner (Fig. 6). ESL milk samples of good quality (furosine $<40 \mathrm{mg} \cdot 100 \mathrm{~g}^{-1}$ protein; $45 \%$ ) were also subdivided into four groups, taking into account the additional limits of 20,15 , and $12 \mathrm{mg} \cdot 100 \mathrm{~g}^{-1}$ protein. Although a furosine content of $20 \mathrm{mg} \cdot 100 \mathrm{~g}^{-1}$ protein had been suggested as upper limit for high pasteurization $[3,4]$, a limit of $40 \mathrm{mg} \cdot 100 \mathrm{~g}^{-1}$ was defined in this study, because of the acid-soluble $\beta$-Lg contents of the respective ESL milk samples of good quality. Nevertheless, the reported upper furosine limit of $20 \mathrm{mg} \cdot 100 \mathrm{~g}^{-1}$ protein for high pasteurization would very nicely fit to the results obtained in the present study, because there were only seven ESL milk samples in between 20 and $40 \mathrm{mg} \cdot 100 \mathrm{~g}^{-1}$ protein. Moreover, five samples thereof were protein-enriched ESL milk, and only two samples (Nos. 49 and 18) had furosine contents of 24.5 and $23.6 \mathrm{mg} \cdot 100 \mathrm{~g}^{-1}$ protein, respectively (Tab. I). Correspondingly, acid-soluble $\beta$-Lg contents were also very low in these two samples (1827 and $1801 \mathrm{mg} \cdot \mathrm{L}^{-1}$ ).

Obviously, ESL milk samples of poor quality had been manufactured using an indirect UHT process that had been reported to be cheaper (regarding investment and production costs) and more energy-efficient compared to the direct UHT heating techniques using injection or infusion systems. On the other hand, directly UHT-treated ESL milk suffers less heat damage than indirectly heated milk and can therefore be used in the production of ESL milk as an alternative to modern microfiltration techniques [10, 15, 18, 24, 25].

\section{CONCLUSIONS}

As ESL milk has shown a dramatic increase in Austria recently, and has been 
widely accepted in many other European countries (e.g. Germany) in the meantime, the nutritional and organoleptic quality of this new category of liquid milk has to be controlled in the future. Since dairy companies are obviously not aware of the negative effects caused by an overheating of liquid milk, there is an urgent need for establishing obligatory threshold levels (limits) for ESL milk regarding TTIs (e.g. acid-soluble $\beta$ - $\mathrm{Lg}$, furosine, and lactulose). In any case, ESL milk should represent a milk product with taste and nutritional quality similar to pasteurized milk, but show the obvious benefits of longer shelf life. However, it must not be as highly heat-treated as UHT milk to fulfil the consumer's expectations regarding nutritional quality of this upcoming product. In striking contrast to these requirements, the present study showed that $55 \%$ of the analysed ESL milk samples had low acid-soluble $\beta$-Lg $\left(<500 \mathrm{mg} \cdot \mathrm{L}^{-1}\right)$ and high furosine contents $\left(>40 \mathrm{mg} \cdot 100 \mathrm{~g}^{-1}\right.$ protein), which was comparable to the excessive heat load of UHT milk. Thus, there is an urgent need for an EU regulation to define obligatory limits for the tolerable heat load of ESL milk, which should be checked by regulatory authorities as soon as possible. In conclusion, results of the present study strongly support the proposed furosine content of $20 \mathrm{mg} \cdot 100 \mathrm{~g}^{-1}$ protein as an upper heating limit for high-pasteurized ESL milk in general $[3,4,6]$, whereas a higher limit of $40 \mathrm{mg} \cdot 100 \mathrm{~g}^{-1}$ protein must be accepted for protein-enriched ESL milks. Nevertheless, acid-soluble $\beta-\mathrm{Lg}$ proved to be the more robust heat indicator for the assessment of heat load of unknown ESL milk samples, and the suggested $\beta-\mathrm{Lg}$ content of $1800 \mathrm{mg} \cdot \mathrm{L}^{-1}$ (or maybe $2000 \mathrm{mg} \cdot \mathrm{L}^{-1}$ ) milk is therefore highly recommended as an upper heating limit for high-pasteurized ESL milk. Hereby, electrophoresis of whey proteins and HPLC of acid-soluble $\beta$-Lg and furosine offer highthroughput, cost-effective, and reliable tools to evaluate and control the heat load of ESL milk to minimize the loss of nutritional quality of milk with ESL in future.

Acknowledgement: The technical assistance of Mrs. Iris Biedermann with the electrophoretic studies is gratefully acknowledged.

\section{REFERENCES}

[1] Anema S.G., Lloyd R.J., Analysis of whey protein denaturation: A comparative study of alternative methods, Milchwissenschaft 54 (1999) 206-210.

[2] Cattaneo S., Masotti F., Pellegrino L., Effects of overprocessing on heat damage of UHT-milk, Eur. Food Res. Technol. 226 (2008) 1099-1106.

[3] Claeys W.L., Smout C., Van Loey A.M., Hendrickx M.E., From time integrator kinetics to time temperature integrator tolerance levels: heat-treated milk, Biotechnol. Prog. 20 (2004) 1-12.

[4] Claeys W.L., Van Loey A.M., Hendrickx M.E., Intrinsic time temperature integrators for heat treatment of milk, Trends Food Sci. Technol. 13 (2002) 293-311.

[5] Claeys W.L., Van Loey A.M., Hendrickx M.E., Kinetics of hydroxymethylfurfural, lactulose and furosine formation in milk with different fat content, J. Dairy Res. 70 (2003) 85-90.

[6] Clawin-Rädecker I., Kiesner C., Martin D., Furosine and ribonucleosides: indicators for the heat treatment of milk, Milchwissenschaft 55 (2000) 679-682.

[7] Delgado T., Corzo N., Santa-Amria G., Jimeno M.N., Olano A., Determination of furosine in milk samples by ion-pair reversed-phase liquid chromatography, Chromatographia 33 (1992) 374-376.

[8] Delgado-Andrade C., Rufián-Henares J.A., Morales F.J., Lysine availability is diminished in commercial fibre-enriched breakfast cereals, Food Chem. 100 (2007) 725-731.

[9] Dyck B., Neue Marktchancen durch ESLTechnologie, Dt. Molk. Ztg. (dmz) 20 (2004) 22-25.

[10] Elliott A.J., Datta N., Amenu B., Deeth H.C., Heat-induced and other chemical changes in commercial UHT milks, J. Dairy Res. 72 (2005) 442-446. 
[11] Erbersdobler H.F., Somoza V., Forty years of furosine - forty years of using Maillard reaction products as indicators of the nutritional quality of foods, Mol. Nutr. Food Res. 51 (2007) 423-430.

[12] FIL/IDF, Standard 20-1, Milk - Determination of Nitrogen Content - Part 1: Kjeldahl Method, Int. Dairy Fed., Brussels, Belgium, 2001.

[13] FIL/IDF, Standard 193, Milk and Milk Products - Determination of Furosine Content - Ion-pair Reverse-phase Highperformance Liquid Chromatography Method, Int. Dairy Fed., Brussels, Belgium, 2004.

[14] FIL/IDF, Standard 178, Liquid Milk Determination of Acid-soluble $\beta$-lactoglobulin Content - Reverse-phased HPLC Method, Int. Dairy Fed., Brussels, Belgium, 2005.

[15] Gallmann P., Eberhard P., Sieber R., Vor- und Nachteile der ESL (Extended Shelf Life)-Milch, Agrarforschung 8 (2001) 112-117.

[16] Guerra-Hernández E., Corzo N., Furosine determination in baby cereals by ion-pair reversed-phase liquid chromatography, Cereal Chem. 73 (1996) 729-731.

[17] Havea P., Singh H., Creamer L.K., Characterization of heat induced aggregates of $\beta$-lactoglobulin, $\alpha$-lactalbumin and bovine serum albumin in a whey protein concentrate environment, J. Dairy Res. 68 (2001) 483-497.

[18] Kaufmann V., Kulozik U., Verfahrenskonzepte zur Herstellung von ESL-Milch, Dt. Milchwirtsch. 58 (2007) 268-271.

[19] Mayer H.K., Bonaparte C., Newart M., Kneifel W., Authentication of Probiotic Bifidobacteria Using Protein and DNA Fingerprinting Techniques, Bulletin Special Issue - IDF Seminar on Fermented Milk,
Special Issue 0301, Int. Dairy Fed., Brussels, Belgium, 2003, pp. 62-84.

[20] Mendoza M.R., Olano A., Villamiel M., Chemical indicators of heat treatment in fortified and special milks, J. Agric. Food Chem. 53 (2005) 2995-2999.

[21] Morales F.J., Romero C., Jiménez-Pérez S., Characterization of industrial processed milk by analysis of heat-induced changes, Int. J. Food Sci. Technol. 35 (2000) 193-200.

[22] Pellegrino L., Resmini P., Luf W., Assessment (indices) of heat treatment of milk, in: Fox P.F. (Ed.), Heat-induced Changes in Milk, 2nd edn., IDF Special Issue 9501, Int. Dairy Fed., Brussels, Belgium, 1995, pp. 409-453.

[23] Resmini P., Pellegrino L., Battelli G., Accurate quantification of furosine in milk and dairy products by a direct HPLC method, Ital. J. Food Sci. 3 (1990) 173-183.

[24] Rysstad G., Kolstad J., Extended shelf life milk - advances in technology, Int. J. Dairy Technol. 59 (2006) 85-96.

[25] Schwermann S., Schwenzow U., Verfahrenskonzepte zur Herstellung von ESLMilch, Dt. Milchwirt. 59 (2008) 384-391, 428-432, 462-467.

[26] Serrano M.A., Castillo G., Muňoz M.M., Hernández A., Influence of hydrolysis, purification, and calibration method on furosine determination using ion-pair reversed-phase high-performance liquid chromatography, J. Chromatogr. Sci. 40 (2002) 87-91.

[27] Tokuşoğlu Ö., Akalin A.S., Unal M.K., A rapid high performance liquid chromatographic detection of furosine ( $\varepsilon-\mathrm{N}-2$-furoylmethyl-L-lysine) in pasteurized and UHT milks, Milchwissenschaft 59 (2004) 502-505. 